\title{
$L_{p}$ Error estimate for minimal norm SBF interpolation
}

\author{
Jianjun Wang ${ }^{1 *}$, Chan-Yun Yang ${ }^{2}$ and Zhigang Gu ${ }^{1}$
}

"Correspondence: wjjmath@gmail.com

${ }^{1}$ School of Mathematics and Statistics, Southwest University, Chongqing, 400715, China Full list of author information is available at the end of the article

\begin{abstract}
By the method of spherical splitting, the interpolation capability of the spherical basis function (SBF) is investigated. As the main result, we deduce the error estimate for the minimal norm SBF interpolation in the metric of the pth Lebesgue integral function space on the sphere. The result shows that the interpolation capability of SBF depends not only on the smoothness of the target function, but also on the geometric distributions of the interpolation knots.
\end{abstract}

MSC: $41 \mathrm{~A} 36 ; 41 \mathrm{~A} 25$

Keywords: spherical basis function; minimal norm; $L_{p}$ approximation; error estimate

\section{Introductions and main results}

Over the past decades, research in the field of function approximation of scattered data points gradually drifted from the polynomials to the radial basis functions (RBFs), and later to the spherical basis functions (SBFs) in a spherical coordinate. Recently, people, such as Wang and Li [1], Freeden et al. [2], and Muller [3], have moved their interests further to the topics of spherical approximation [1-7]. Based on the developments, spherical harmonic analysis was established and had some considerable progress. Meanwhile, $L_{p}$ error estimations for the SBF approximation were studied hereafter and stepped forward in the studies of Le Gia et al. [4], Hubbert and Morton [5], and Sloan and Wendland [7] after 2004. In 2007, Chen [8] established error bound for the minimal norm interpolation on a sphere. With this plentiful foundation, Lin and Cao [9] embedded firstly the smooth SBFs in a native space and specified the error bound between the best approximation and the target function via $L_{p}$ metric. As a consecutive study, the paper thus aims to derive a minimal norm interpolation with the $L_{p}$ measure.

For a fixed integer $q(q \geq 1), S^{q}$ is a unit sphere in $R^{q+1}$, i.e., $S^{q}=\left\{x=\left(x_{1}, x_{2}, \ldots, x_{q+1}\right) \in\right.$ $\left.R^{q+1}, x_{1}^{2}+x_{2}^{2}+\cdots+x_{q+1}^{2}=1\right\}$, and $d \omega$ represents a sufficient small elemental area on the spherical surface $S^{q}$. The total surface area of $S^{q}$ can hence be expressed as

$$
\omega_{q}=\int_{S^{q}} d \omega=\frac{2 \pi^{\frac{q+1}{2}}}{\Gamma\left(\frac{q+1}{2}\right)} .
$$

In regard to $d \omega$, the $L^{p}\left(S^{q}\right)$ inner product of functions $f$ and $g$ is given as

$$
(f, g)_{L^{p}\left(S^{q}\right)}=\int_{S^{q}} f(x) g(x) d \omega(x)
$$

○2013 Wang et al:; licensee Springer. This is an Open Access article distributed under the terms of the Creative Commons Attribution License (http://creativecommons.org/licenses/by/2.0), which permits unrestricted use, distribution, and reproduction in any medium, provided the original work is properly cited. 
and the $L^{p}\left(S^{q}\right)$ norm is defined as

$$
\|f\|_{p}:=\|f\|_{L^{p}\left(S^{q}\right)}:= \begin{cases}\left(\int_{S^{q}}|f(x)|^{p} d \omega\right)^{\frac{1}{p}}, & 1 \leq p<+\infty \\ \max _{x \in S^{q}}|f(x)|, & p=+\infty,\end{cases}
$$

where $L^{p}\left(S^{q}\right)$ represents a function space constructed by a complex function, $f: S^{q} \rightarrow C$, fulfilling $\|f\|_{p}<+\infty$. An identical function in $L^{p}\left(S^{q}\right)$ is characterized as a function identical to the functions which have the same output values everywhere with the same inputs.

For an integer $k \geq 0, H_{k}^{q}$ denotes a linear subspace in $R^{q+1}$ constructed by all the $k$ th order homogeneous harmonic polynomials $p(x)$ restricted by $S^{q}$, and $\Pi_{n}^{q}$ denotes the function constructed by all the $k$ th order, $k \leq n$, spherical harmonic polynomials. The relationship between $\Pi_{n}^{q}$ and $H_{k}^{q}$ can primarily be given as

$$
\Pi_{n}^{q}=\bigoplus_{k=0}^{n} H_{k}^{q}
$$

Obviously, the dimension of $H_{k}^{q}$ is

$$
d_{k}^{q}=\operatorname{dim} H_{k}^{q}= \begin{cases}\frac{2 k+q-1}{k+q-1}\left(\begin{array}{c}
2 k+q-1 \\
k+q-1
\end{array}\right), & k \geq 1 \\
1, & k=0 .\end{cases}
$$

In $H_{k}^{q}$, we select a set of functions $Y_{k, j}\left(j=1,2, \ldots, d_{k}^{q}\right)$ to form a standard orthogonal basis $\Pi_{n}^{q}=\left\{Y_{k, j}, j=1,2, \ldots, d_{k}^{q}, k=0,1,2, \ldots, n\right\}$. The subspace $H_{k}^{q}$ also confirms $L^{2}\left(S^{q}\right)=$ $\bigoplus_{k=0}^{\infty} H_{k}^{q}$ and the famous additive law:

$$
\sum_{j=1}^{d_{k}^{q}} Y_{k, j}(\xi) Y_{k, j}(\eta)=\frac{d_{k}^{q}}{\omega_{q}} P_{k}(q+1: \xi \cdot \eta)
$$

where $\xi \cdot \eta$ denotes the inner product of $\xi$ and $\eta . P_{k}(q+1: x)$ denotes the $k$ th order Legendre polynomial satisfying $P_{k}(q+1: 1)=1$ and complies with

$$
\int_{-1}^{1} P_{k}(q+1: x) P_{l}(q+1: x)\left(1-x^{2}\right)^{\frac{q}{2}-1} d x=\frac{\omega_{q}}{\omega_{q-1} d_{k}^{q}} \delta_{k, l}
$$

By giving $A=\left\{\left\{A_{n, j}\right\}: A_{n, j} \in R, n=0,1,2, \ldots, j=1, \ldots, d_{n}^{q}\right\}$, a series $\left\{A_{n}\right\} \in A, A_{n}>0, n=$ $0,1,2, \ldots$, can also be expressed as $\left\{A_{n, j}\right\} \in A, A_{n, j}=A_{n}, j=1, \ldots, d_{n}^{q}$. Using the series $\left\{A_{n, j}\right\}$, a space defined as

$$
H^{s}\left(S^{q}\right)=\left\{F \in L^{2}\left(S^{q}\right), \sum_{n=0}^{\infty} \sum_{j=1}^{d_{n}^{q}} A_{n}^{s}\left(F, Y_{n, j}\right)^{2}<\infty\right\}
$$

has the inner product $(\cdot, \cdot)_{H^{s}\left(S^{q}\right)}$ :

$$
(F, G)_{H^{s}\left(S^{q}\right)}=\sum_{n=0}^{\infty} \sum_{j=1}^{d_{n}^{q}} A_{n}^{s}\left(F, Y_{n, j}\right)\left(G, Y_{n, j}\right),
$$


and the corresponding norm is consequently defined as

$$
\|F\|_{H^{s}\left(S^{q}\right)}=\left((F, F)_{H^{s}\left(S^{q}\right)}\right)^{\frac{1}{2}} .
$$

As known from the definition here, $H^{s}\left(S^{q}\right)$ is a Hilbert space, i.e., when $s>\frac{q}{2}, H^{s}\left(S^{q}\right)$ has a reproducing kernel

$$
K(\xi, \eta)=\sum_{n=0}^{\infty} \sum_{j=1}^{d_{n}^{q}} A_{n}^{-s} Y_{n, j}(\xi) Y_{n, j}(\eta)
$$

if $A_{n}$ satisfies $A_{n} \sim 1+n^{2}$ (see the reference [7]).

Hereafter, we assume consistently in this study that $H^{s}\left(S^{q}\right)$ is a reproducing kernel Hilbert space (RKHS) constructed by the reproducing kernel and call the space a native space of $K(\xi, \eta)$ (see $[10,11])$. From the additive law, we have

$$
K(\xi, \eta)=\sum_{n=0}^{\infty} \sum_{j=1}^{d_{n}^{q}} A_{n}^{-s} Y_{n, j}(\xi) Y_{n, j}(\eta)=\sum_{n=0}^{\infty} \frac{d_{n}^{q}}{\omega_{q}} A_{n}^{-s} P_{n}(q+1, \xi \cdot \eta), \quad \xi, \eta \in S^{q}
$$

where $P_{n}(q+1, x)$ is the $n$th order Legendre polynomial. Here $K(\xi, \eta)$ is obviously a spherical radial basis function.

Let us assume that $X=\left\{x_{1}, x_{2}, \ldots, x_{N}\right\}$ is a set of $N$ points taken from the unit sphere $S^{q}$ and has $N$ output values $y_{1}, y_{2}, \ldots, y_{N}$ corresponding to $x_{1}, x_{2}, \ldots, x_{N}$ through a function $F$ :

$$
I_{N}(y)=\left\{F \in H^{s}\left(S^{q}\right): F\left(x_{i}\right)=y_{i}, i=1,2, \ldots, N\right\} .
$$

If there exists $F \in I_{N}(y)$ such that $\|F\|$ can be minimized, we say that the minimization of $F$ is a problem subject to a minimal norm. By denoting $S_{N}(\xi)$ as an interpolation with the minimal norm, there is a kernel basis expression for the interpolation $S_{N}(\xi)$ (see the references $[2,7,8])$ :

$$
S_{N}(\xi)=\sum_{i=0}^{N} \alpha_{i} K\left(x_{i}, \xi\right), \quad \xi \in S^{q}
$$

Suppose that $y_{i}, i=1,2, \ldots, N$, are produced by the function $f(x)$, i.e., $f\left(x_{i}\right)=y_{i}, i=$ $1, \ldots, N$. The interpolation problem becomes intrinsically a function approximation problem to estimate the error between $f(x)$ and $S_{N}(x)$. The approximation order of the interpolation can then be determined by the grid norm, $h$, and the input $x_{i} \in X$. Here, $h$ is defined as

$$
h:=\sup _{x \in S^{q}} \min _{i} \in X \operatorname{dist}\left(x, x_{i}\right)
$$

where $\operatorname{dist}(\xi, \eta)$ is the spherical distance between $\xi$ and $\eta$, i.e., $\operatorname{dist}(\xi, \eta)=\arccos (\xi, \eta)$, $\xi, \eta \in S^{q}$. 
Because $H^{s}\left(S^{q}\right)$ is a RKHS constituted by the reproducing kernel $K(\xi, \eta)$, the error between $f(x)$ and $S_{N}(x)$ in the knot $x_{i}$ will vanish, i.e.,

$$
\left(S_{N}-f, K\left(\cdot, x_{i}\right)\right)_{H^{s}\left(S^{q}\right)}=S_{N}\left(x_{i}\right)-f\left(x_{i}\right)=0
$$

for an arbitrary $f \in H^{s}\left(S^{q}\right)$, if $S_{N}$ is interpolated via the minimal norm.

Suppose $V_{x}:=\operatorname{span}\left\{K\left(\cdot, x_{1}\right), \ldots, K\left(\cdot, x_{N}\right)\right\}$, we thus have

$$
\left(S_{N}-f, v\right)_{H^{s}\left(S^{q}\right)}=0
$$

for a given arbitrary $v \in V_{x}$. It implies that $S_{N}$ is an orthogonal projection of $f$ to the span $V_{x}$ in the space $H^{s}\left(S^{q}\right)$.

Furthermore, the $L^{p}(p=+\infty)$ approximation of an interpolation via minimal norm is consequently bounded as follows. By assuming there exists a positive integer $m$ such that the grid norm $h \leq \frac{1}{2 m}$ for $X=\left\{x_{1}, x_{2}, \ldots, x_{N}\right\}$ is taken from $S^{q}$, we deduce that the $L^{p}$ $(p=+\infty)$ approximation via the minimal norm must satisfy

$$
\sup _{x \in S^{q}}\left|f(x)-S_{N}(x)\right| \leq C\left(\sum_{n=m+1}^{\infty} \frac{d_{n}^{q}}{A_{n}^{2}}\right)\|f\|
$$

for an arbitrary $f \in H^{s}\left(S^{q}\right)$ (refer to [8]). Coming with these contributions, this study is sought to extend the significant results to a more general case of $L^{p}(1 \leq p \leq+\infty)$.

Theorem 1.1 Assume that $X=\left\{x_{1}, x_{2}, \ldots, x_{N}\right\}$ is a set of $N$ points taken from the unit sphere $S^{q}, h$ is the corresponding grid norm, and there exists a positive integer $m$ such that $h \leq \frac{1}{2 m}$. For an arbitrary function $f \in H^{s}\left(S^{q}\right), s>\frac{q}{2}$, and its corresponding interpolation via the minimal norm $S_{N}$, there must exist a positive constant $C$, which is independent off and $h$, such that the following estimations are satisfied:

$$
\begin{aligned}
\left\|f-S_{N}\right\|_{p} & \leq C h^{s+\frac{q}{p}-\frac{q}{2}}\left(\sum_{n=m+1}^{\infty} \frac{d_{n}^{q}}{A_{n}^{2}}\right)^{\frac{1}{2}}\left\|f-S_{N}\right\|_{H^{s}\left(S^{q}\right)} \\
& \leq C h^{s+\frac{q}{p}-\frac{q}{2}}\left(\sum_{n=m+1}^{\infty} \frac{d_{n}^{q}}{A_{n}^{2}}\right)^{\frac{1}{2}}\|f\|_{H^{s}\left(S^{q}\right)}, \quad 2 \leq p<\infty
\end{aligned}
$$

and

$$
\begin{aligned}
\left\|f-S_{N}\right\|_{p} & \leq C h^{s}\left(\sum_{n=m+1}^{\infty} \frac{d_{n}^{q}}{A_{n}^{2}}\right)^{\frac{1}{2}}\left\|f-S_{N}\right\|_{H^{s}\left(S^{q}\right)} \\
& \leq C h^{s}\left(\sum_{n=m+1}^{\infty} \frac{d_{n}^{q}}{A_{n}^{2}}\right)^{\frac{1}{2}}\|f\|_{H^{s}\left(S^{q}\right)}, \quad 1 \leq p<2 .
\end{aligned}
$$

Theorem 1.2 Assume that $X=\left\{x_{1}, x_{2}, \ldots, x_{N}\right\}$ is a set of $N$ points taken from the unit sphere $S^{q}$, and the grid norm satisfies the condition $h \leq \frac{1}{2 m}$. For an arbitrary function $f \in H^{s}\left(S^{q}\right)$, 
$s>\frac{q}{2}$, and its corresponding interpolation via the minimal norm $S_{N}$, we have

$$
\left\|f-S_{N}\right\|_{p} \leq C h^{2 s+\frac{q}{p}-\frac{q}{2}}\left(\sum_{n=m+1}^{\infty} \frac{d_{n}^{q}}{A_{n}^{2}}\right)^{\frac{1}{2}}\|f\|_{H^{2 s}\left(S^{q}\right)}, \quad 2 \leq p<\infty
$$

and

$$
\left\|f-S_{N}\right\|_{p} \leq C h^{2 s}\left(\sum_{n=m+1}^{\infty} \frac{d_{n}^{q}}{A_{n}^{2}}\right)^{\frac{1}{2}}\|f\|_{H^{2 s}\left(S^{q}\right)}, \quad 1 \leq p<2,
$$

where $C$ denotes a positive constant independent of $f$ and $h$.

To prove the main results of Theorems 1.1 and 1.2, the concept of spherical cap should be introduced first: With center $x_{0}$ and radius $\gamma$, the spherical cap $G\left(x_{0}, \gamma\right)$ is defined as

$$
G\left(x_{0}, \gamma\right)=\left\{x \in S^{q}, x \cdot x_{0}>\cos \gamma\right\}
$$

and the surface area, referred to as $G(\gamma)$ of $G(x, \gamma)$, can be given as

$$
G(\gamma)=\int_{0}^{\gamma} \omega_{q} \sin ^{q-1} \theta d \theta
$$

\section{Lemmas}

To completely prove Theorems 1.1 and 1.2, five lemmas are given as follows.

Lemma 2.1 (refer to $[5,6,9]$ ) Assume an integer $q \geq 1$, constants $M=2 \sqrt{q}$ and $\delta_{q}=$ $\frac{1}{(4 q+1)^{\frac{3}{2}}}$, and $h_{0}:=\frac{\theta}{M+M_{1}+\delta_{q}}$ with an arbitrary positive number $M_{1}$, and $\theta \in\left(0, \frac{\pi}{3}\right)$, then there exists a point set $Z_{h} \subset S^{q}$ with an arbitrary $h \in\left(0, h_{0}\right)$ satisfying

$$
S^{q}=\bigcup_{z \in Z_{h}} G(z, M h)
$$

If $F_{A}$ represents the characteristic function of a given set $A \in S^{q}$, there exists a positive integer $Q$ independent of h satisfying

$$
\sum_{z \in Z_{h}} F_{G(z, \bar{M} h)} \leq Q
$$

where $\bar{M}=M+M_{1}$. Furthermore, there exists a constant $C_{Q}$ independent of $h$ such that $\left|Z_{h}\right| \leq C_{Q} h^{-q}$.

Lemma 2.2 (refer to $[5,6,9]$ ) By giving constants $S \geq 0, M_{1}, C, \bar{M}=M+M_{1}, h_{0}=\frac{C}{3 \bar{M}}$ and $h \in\left(0, h_{0}\right)$, there must exist an arbitrary $f \in H^{s}\left(S^{q}\right)$ such that

$$
\sum_{z \in Z_{h}}\|f\|_{H^{s}(G(z, \bar{M} h))}^{2} \leq Q\|f\|_{H^{s}\left(S^{q}\right)}^{2} .
$$

Lemma 2.3 Assume that $\eta_{1}^{*}$ is an evaluation functional corresponding to $\eta_{1}$ in $H^{s}\left(S^{q}\right), \eta_{1}^{*}$ in $H^{s}\left(S^{q}\right)$ can alternatively be expressed as $K\left(\eta_{1}, \xi\right)$. 
Lemma 2.4 Assume that $x^{*}, x_{1}^{*}, x_{2}^{*}, \ldots, x_{N}^{*}$ are evaluation functionals in $H^{s}\left(S^{q}\right)$ and their expressions in $H^{s}\left(S^{q}\right)$ are $u, u_{1}, \ldots, u_{N}$. Together with $S_{N}$, which is interpolated via the minimal norm along $x_{1}, x_{2}, \ldots, x_{N}$, and $z$, which is the optimized approximation of $u$ in the $\operatorname{span}\left\{u_{1}, u_{2}, \ldots, u_{N}\right\}$, we have

$$
\left|f(x)-S_{N}(x)\right| \leq\|u-z\|\left\|f-s_{N}\right\|_{2} .
$$

It should be noted that Lemmas 2.3 and 2.4 can be directly obtained from [9].

Lemma 2.5 (refer to $[4,5,7])$ There exist two constants $C>0$ and $h_{1}>0$, dependent only on $s$ and $d$, such that a function $g\left(g \in H^{s}\left(S^{q}\right),\left.g\right|_{X}=0\right.$ for arbitrary $\left.X \in S^{d}, h \leq h_{1}\right)$ satisfies

$$
\|g\|_{2} \leq C h^{s}\|g\|_{H^{s}(S q)},
$$

when $s>\frac{d}{2}$.

\section{Proof of theorems}

Proof of Theorem 1.1 From Lemma 2.1, we have

$$
\begin{aligned}
\left\|f-S_{N}\right\|_{p}^{p} & =\int_{S^{q}}\left|\left(f-S_{N}\right)(\xi)\right|^{p} d \omega(\xi) \\
& \leq \sum_{z \in Z_{h}} \int_{G(z, M h)}\left|\left(f-S_{N}\right)(\xi)\right|^{p} d \omega(\xi)
\end{aligned}
$$

for arbitrary $1 \leq p<\infty$ and $M=2 \sqrt{q}$. We consider first the local error estimation. Since $f-S_{N}$ is continuous on $\overline{G(z, M h)}$, and $\overline{G(z, M h)}$ is a compact support of $S^{q}$, there must exist $\xi_{z} \in \overline{G(z, M h)}$ such that $f-S_{N}$ is maximized at $\xi_{z}$. We consequently have

$$
\begin{aligned}
\left\|f-S_{N}\right\|_{p}^{p} & \leq \sum_{z \in Z_{h}}\left|\left(f-S_{N}\right)\left(\xi_{z}\right)\right|^{p} \int_{G(z, M h)} d \omega(\xi) \\
& \leq C_{q} h^{q} \sum_{z \in Z_{h}}\left|\left(f-S_{N}\right)\left(\xi_{z}\right)\right|^{p},
\end{aligned}
$$

where $C_{q}$ is a constant dependent only on $q$ and satisfying $G(M h)=C_{q} h^{q}$.

(a) When $p \geq 2$ : Taking $\xi_{z}^{*}$ as an evaluation functional, $S_{\xi_{z}}^{*}$ is its best approximation in $\operatorname{span}\left\{u_{1}, u_{2}, \ldots, u_{N}\right\}$. From the Jensen inequality (refer to $\left.[9,12]\right), \sum_{i=1}^{N} a_{i}^{p} \leq\left(\sum_{i=1}^{N} a_{i}^{2}\right)^{\frac{p}{2}}$, Lemmas 2.3 and 2.4, we obtain

$$
\begin{aligned}
\left\|f-S_{N}\right\|_{p}^{p} & \leq C h^{q}\left(\sum_{z \in Z_{h}}\left|\left(f-S_{N}\right)\left(\xi_{z}\right)\right|^{2}\right)^{\frac{p}{2}} \\
& \leq C h^{q}\left(\sum_{z \in Z_{h}}\left\|\xi_{z}^{*}-S_{\xi_{z}}^{*}\right\|^{2}\left\|f-S_{N}\right\|_{L^{2}(G(z, M h))}^{2}\right)^{\frac{p}{2}} \\
& \leq C h^{q}\left(\sum_{z \in Z_{h}}\left\|f-S_{N}\right\|_{L^{2}(G(z, M h))}^{2}\left\|K\left(\xi_{z}, \cdot\right)-\sum_{i=1}^{N} \alpha_{i} K\left(x_{i}, \cdot\right)\right\|^{2}\right)^{\frac{p}{2}}
\end{aligned}
$$




$$
\begin{aligned}
& \leq C h^{q}\left(\sum_{z \in Z_{h}}\left\|f-S_{N}\right\|_{L^{2}(G(z, M h))}^{2}\right)^{\frac{p}{2}}\left(\sum_{z \in Z_{h}}\left\|K\left(\xi_{z}, \cdot\right)-\sum_{i=1}^{N} \alpha_{i} K\left(x_{i}, \cdot\right)\right\|^{2}\right)^{\frac{p}{2}} \\
& \leq C h^{q}\left(\sum_{z \in Z_{h}}\left\|f-S_{N}\right\|_{L^{2}(G(z, M h))}^{2}\right)^{\frac{p}{2}}\left(\sum_{z \in Z_{h}} \sum_{n=m+1}^{\infty} \frac{d_{n}^{q}}{A_{n}^{2}}\right)^{\frac{p}{2}} .
\end{aligned}
$$

It should be noted that one of the following inequalities (refer to [8]) is used in the last step of the derivations above

$$
\left\|K(x, \cdot)-\sum_{i=1}^{N} \alpha_{i} K\left(x_{i}, \cdot\right)\right\|^{2} \leq C \sum_{n=m+1}^{\infty} \frac{d_{n}^{q}}{A_{n}^{2}} .
$$

For sure, by taking $\overline{h_{0}}=\frac{1}{2 m}$ (where $\left.\overline{h_{0}}=\min \left\{h_{0}, h_{1}\right\}\right)$, we can go further

$$
\begin{aligned}
\left\|f-S_{N}\right\|_{p}^{p} & \leq C h^{q+s p}\left(\sum_{z \in Z_{h}}\left\|f-S_{N}\right\|_{H^{s}(G(z, M h))}^{2}\right)^{\frac{p}{2}}\left(\sum_{n=m+1}^{\infty} \frac{d_{n}^{q}}{A_{n}^{2}}\right)^{\frac{p}{2}} C_{Q} h^{-\frac{p q}{2}} \\
& \leq C h^{q+s p-\frac{p q}{2}}\left(\sum_{n=m+1}^{\infty} \frac{d_{n}^{q}}{A_{n}^{2}}\right)^{\frac{p}{2}}\left\|f-S_{N}\right\|_{H^{s}\left(S^{q}\right)}^{p}
\end{aligned}
$$

with $h \in\left(0, \overline{h_{0}}\right)$. By using both $M<\bar{M}$ and the consequence $\left|Z_{h}\right| \leq C_{Q} h^{-q}$, Lemma 2.5, Lemma 2.2, and Lemma 2.1, we achieve

$$
\left\|f-S_{N}\right\|_{p} \leq C h^{s+\frac{q}{p}-\frac{q}{2}}\left(\sum_{n=m+1}^{\infty} \frac{d_{n}^{q}}{A_{n}^{2}}\right)^{\frac{1}{2}}\left\|f-S_{N}\right\|_{H^{s}\left(S^{q}\right)} .
$$

(b) When $1 \leq p<2$ : From the inequality (refer to $[9,12]$ )

$$
\sum_{i=1}^{N} a_{i}^{p} \leq N^{1-\frac{p}{2}}\left(\sum_{i=1}^{N} a_{i}^{2}\right)^{\frac{p}{2}}
$$

and the fact which $Z_{h}$ is less than $C h^{-q}$, we obtain first

$$
\begin{aligned}
\left\|f-S_{N}\right\|_{p}^{p} & \leq C h^{q}\left(\sum_{z \in Z_{h}}\left|\left(f-S_{N}\right)\left(\xi_{z}\right)\right|^{2}\right)^{\frac{p}{2}} h^{-\left(1-\frac{p}{2}\right) q} \\
& \leq C h^{\frac{p q}{2}}\left(\sum_{z \in Z_{h}}\left\|\xi_{z}^{*}-S_{\xi_{z}}^{*}\right\|^{2}\left\|f-S_{N}\right\|_{L^{2}(G(z, M h))}^{2}\right)^{\frac{p}{2}} \\
& \leq C h^{s p}\left(\sum_{n=m+1}^{\infty} \frac{d_{n}^{q}}{A_{n}^{2}}\right)^{\frac{p}{2}}\left\|f-S_{N}\right\|_{H^{s}\left(S^{q}\right)}^{p} .
\end{aligned}
$$

Following similarly the steps in the case of $p \geq 2$, we also have

$$
\left\|f-S_{N}\right\|_{p} \leq C h^{s}\left(\sum_{n=m+1}^{\infty} \frac{d_{n}^{q}}{A_{n}^{2}}\right)^{\frac{1}{2}}\left\|f-S_{N}\right\|_{H^{s}(S q)} .
$$


With the fact that $S_{N}$ is the orthogonal projection of $f$ in $H^{s}\left(S^{q}\right)$, the proof of Theorem 1.1 is thus completed.

Proof of Theorem 1.2 From the property of orthogonal projection of $S_{N}$, the CauchySchwarz inequality, and Lemma 2.5 , we can easily obtain

$$
\begin{aligned}
\left\|f-S_{N}\right\|_{H^{s}\left(S^{q}\right)}^{p} & =\left(\left\|f-S_{N}\right\|_{H^{s}\left(S^{q}\right)}^{2}\right)^{\frac{p}{2}} \\
& =\left(f-S_{N}, f-S_{N}\right)_{H^{s}\left(S^{q}\right)}^{\frac{p}{2}} \\
& =\left(f-S_{N}, f\right)_{H^{s}\left(S^{q}\right)}^{\frac{p}{2}} \\
& =\left(\sum_{n=0}^{\infty} \sum_{j=1}^{d_{n}^{q}} A_{n}^{s}\left(f-S_{N}, Y_{n, j}\right)\left(f, Y_{n, j}\right)\right)^{\frac{p}{2}} \\
& \leq\left(\left(\sum_{n=0}^{\infty} \sum_{j=1}^{d_{n}^{q}} A_{n}^{2 s}\left(f, Y_{n, j}\right)^{2}\right)^{\frac{1}{2}}\left(\sum_{n=0}^{\infty} \sum_{j=1}^{d_{n}^{q}}\left(f-S_{N}, Y_{n, j}\right)^{2}\right)^{\frac{1}{2}}\right)^{\frac{p}{2}} \\
& =\left(\|f\|_{H^{2 s}\left(S^{q}\right)}\left\|f-S_{N}\right\|_{L_{2}\left(S^{q}\right)}\right)^{\frac{p}{2}} \\
& \leq C h^{\frac{s p}{2}}\|f\|_{H^{2 s}\left(S^{q}\right)}^{\frac{p}{2}}\left\|f-S_{N}\right\|_{H^{s}\left(S^{q}\right)}^{\frac{p}{2}}
\end{aligned}
$$

Therefore

$$
\left\|f-S_{N}\right\|_{H^{s}\left(S^{q}\right)}^{p} \leq C h^{s p}\|f\|_{H^{2 s}\left(S^{q}\right)}^{p} .
$$

Together with the results of Theorem 1.1, Theorem 1.2 is thus established.

\section{Competing interests}

The authors declare that they have no competing interests.

\section{Authors' contributions}

In this paper, JW had the main role in providing derivations. CY and ZG also contributed significantly with their corresponding effort to finish the work.

\section{Author details}

'School of Mathematics and Statistics, Southwest University, Chongqing, 400715, China. ${ }^{2}$ Department of Electrical Engineering, National Taipei University, No.151, University Rd., San Shia District, New Taipei City, 23741, Taiwan.

\section{Acknowledgements}

The first author and the third author would like to thank the Natural Science Foundation of China (Nos. 61273020, 11001227), the Fundamental Research Funds for the Central Universities (No. XDJK2010B005).

Received: 25 February 2013 Accepted: 27 August 2013 Published: 08 Nov 2013

\section{References}

1. Wang, KY, Li, LQ: Harmonic Analysis and Approximation on the Unit Sphere. Science Press, Beijing (2000)

2. Freeden, W, Gervens, T, Schreiner, M: Constructive Approximation on the Sphere. Oxford University Press, New York (1998)

3. Müller, C: Spherical Harmonics. Lecture Notes in Mathematics, vol. 17. Springer, New York (1966)

4. Le Gia, QL, Narcowich, F, Ward, J, Wendland, H: Continuous and discrete least-squares approximation by basis functions on spheres. J. Approx. Theory 143, 124-133 (2006)

5. Hubbert, S, Morton, TM: $L_{p}$-Error estimates for radial basis function interpolation on the sphere. J. Approx. Theory 129 58-77 (2004)

6. Hubbert, S, Morton, TM: A Duchon framework for the sphere. J. Approx. Theory 129, 28-57 (2004)

7. Sloan, LH, Wendland, $\mathrm{H}$ : Inf-sup condition for spherical polynomials and radial basis functions on spheres. Math. Comput. 267, 1319-1331 (2009) 
8. Chen, ZX: Error estimates of minimal norm interpolant approximation by the spherical radial basis functions. Acta Math. Appl. Sin. 30(1), 159-167 (2007) (in Chinese)

9. Lin, SB, Cao, FL: $L^{p}$ Approximation by shifts of smooth kernels on spheres. Chin. Ann. Math., Ser. A 31(5), 615-624 (2010) (in Chinese)

10. Wendland, H: Scattered Data Approximation. Cambridge University Press, Cambridge (2005)

11. Narcowich, F, Schaback, R, Ward, J: Approximation in Sobolev spaces by kernel expansions. J. Approx. Theory 114(1), 70-83 (2002)

12. Brenner, SC, Scott, RL: The Mathematical Theory of Finite Element Methods. Springer, New York (1994)

10.1186/1029-242X-2013-510

Cite this article as: Wang et al.: $L_{p}$ Error estimate for minimal norm SBF interpolation. Journal of Inequalities and Applications 2013, 2013:510

Submit your manuscript to a SpringerOpen ${ }^{\circ}$ journal and benefit from:

- Convenient online submission

- Rigorous peer review

Immediate publication on acceptance

- Open access: articles freely available online

- High visibility within the field

- Retaining the copyright to your article 